\title{
La comprensión lectora \\ fortalecida por los textos expositivos ${ }^{*}$
}

\author{
María Elizabeth Bustamante \\ Institución Educativa Miraflores, Medellín, Colombia \\ lizb1504@gmail.com \\ Sara Hernández Moreno \\ Institución Educativa San Pablo, Medellín, Colombia \\ hernandez.sara03@gmail.com \\ María Victoria Restrepo Higuita \\ Institución Educativa Compartir, Medellín, Colombia \\ vicky1068@gmail.com \\ Paola Andrea Ríos Escohar \\ Institución Educativa San Pablo, Medellín, Colombia \\ parios25008@gmail.com
}

\section{RESUMEN}

Este artículo parte de la hipótesis de que a mayor uso de los textos expositivos, mejor es el grado de comprensión lectora de los estudiantes. Se busca resaltar algunos resultados emanados de la investigación Prácticas de lectura con textos expositivos para mejorar la comprensión lectora en los estudiantes de grado $5^{\circ}$, a través de unas secuencias didácticas, en las Instituciones educativas San Pablo y Compartir. La investigación, bajo un enfoque cualita- tivo y con el diseño de las secuencias con estrategias y actividades intencionadas, permitieron establecer algunas consideraciones en torno a los procesos de enseñanza-aprendizaje de la compresión lectora para estudiantes y docentes, y potenciar el trabajo con los textos expositivos.

Palabras clave: comprensión lectora; textos expositivos; secuencias didácticas; grado quinto.

Cómo citar: Bustamante, M. E., Hernández Moreno, S., Restrepo Higuita, M. V. y Ríos Escobar, P. A. (2019). La comprensión lectora fortalecida por los textos expositivos. Ciencias Sociales y Educación, 8(16), 133-147. DOI: https://doi.org/10.22395/csye.v8n16a8

Recibido: 15 de mayo de 2019.

Aprobado: 25 de septiembre de 2019. 


\section{Reading Comprehension Strengthened by Expository Texts}

\section{ABSTRACT}

This article is based on the hypothesis that the greater the use of expository texts the better the reading comprehension level among the students; it aims to highlight some results derived from the research Prácticas de lectura con textos expositivos para mejorar la comprensión lectora en los estudiantes de grado $5^{\circ}$ (Reading practices with expository texts for the improvement of reading comprehension in 5th-grade students) through a series of didactic sequences in the San Pablo and Compartir schools. The research was performed under a qualitative approach and it allowed, through the design of the sequences with a strategy and intentional activities, to establish some considerations around the teaching-learning processes of reading comprehension for students and teachers and thus invigorating the work with expository texts.

Keywords: reading comprehension; expository texts; didactic sequences; fifth grade.

\section{A compreensão leitopa fortalecida pelos textos expositivos}

\section{RESUMO}

Este artigo parte da hipótese de que quanto maior o uso dos textos expositivos, melhor o grau de compreensão leitora dos estudantes. Além disso, pretende destacar alguns resultados da pesquisa "Práticas de leitura com textos expositivos para melhorar a compreensão leitora nos estudantes do $5^{\circ}$ grau", por meio de sequências didáticas, nas instituições educativas San Pablo e Compartir, Colômbia. A pesquisa, de abordagem qualitativa e com desenho das sequências com estratégias e atividades intencionadas, permitiu estabelecer algumas considerações sobre os processos de ensino-aprendizagem da compreensão leitora para estudantes e docentes, e potencializar o trabalho com os textos expositivos.

Palavras-chave: compreensão leitora; textos expositivos; sequências didáticas; quinto grau. 


\section{Introducción}

Los diseños curriculares en Colombia en el área de lengua castellana hacen una apuesta grande a la comprensión lectora, es ella la encargada en las pruebas estandarizadas de medir y evaluar a los estudiantes. El bajo rendimiento de los estudiantes del Valle de Aburrá en las pruebas Saber y el desempeño de las instituciones en las cuales fue realizada la intervención, con promedios muy por debajo de la media nacional, hicieron necesaria la investigación Prácticas de lectura con textos expositivos para mejorar la comprensión lectora en los estudiantes de grado $5^{\circ}$.

Luego de conocer los contextos socioculturales y de revisar el informe detallado de lenguaje, se enunció la problemática que se vivía alrededor de la comprensión lectora en ambas instituciones. Por ello, fue indispensable establecer mecanismos que intervinieran las ausencias en los procesos de comprensión de la información. Las principales razones estaban movidas por un déficit en la decodificación fluida de la lectura. Los estudiantes decodificaban de manera automática pero no comprendían lo que leían debido al uso de metodologías silábicas que se centraban en la lectura de palabras, y en unas orientaciones encaminadas a la lectura recorrida, pero especialmente cuando las pruebas de Estado establecieron tres niveles de lectura: literal, inferencial y críticointertextual, como base para identificar qué tanto sabe un estudiante y qué es capaz de hacer con ese conocimiento.

En este punto se hizo comprensible la necesidad de incentivar el uso de los textos expositivos como mecanismo de aprendizaje transversal para el fortalecimiento de los niveles de lectura, y potenciar los argumentos que desarrollaran el pensamiento crítico dentro de los estándares, lineamientos y currículos. En el currículo de estas instituciones, el texto expositivo solo era una temática a abordar y un logro a cumplir que se sumaba a la gran variedad de tipología textual sin un enfoque pedagógico. Los textos expositivos tienen unas características y una estructura determinada que pueden brindar estrategias que posibiliten el desarrollo de procesos cognoscitivos superiores y que ayudan a mejorar competencias en comprensión e interpretación textual.

Para el proceso se implementaron cuatro fases. En la primera se hizo un diagnóstico o recolección de información, se realizaron entrevistas semiestructuradas aplicadas a ocho docentes de básica primaria del grado quinto de las dos instituciones educativas. Los perfiles de los docentes variaron por su titulación: licenciados, magísteres, normalistas, pertenecientes a las áreas de matemáticas, humanidades, ética, ciencias sociales y ciencias naturales. También una entrevista semiestructurada dirigida a estudiantes para conocer el acercamiento a los textos expositivos y las estrategias que les eran aplica- 
das para su comprensión, y luego una observación no participante de clase a docentes del grado quinto.

Una vez recolectada la información y analizada, la segunda fase consistió en el diseño de las secuencias didácticas. Los momentos que hicieron parte de estas fueron tomados y adaptados desde la propuesta del Ministerio de Educación Nacional.

Según los propósitos de la investigación y el tiempo de ejecución para el alcance de la misma, se intervino con cuatro secuencias didácticas que comprendieron sesiones de sesenta minutos cada una, las cuales contemplaron diferentes áreas y referentes de calidad que sustentaron la pertinencia y objetividad de cada una de ellas.

Diseñadas las actividades, en la tercera fase se pusieron en escena los contextos referidos, se incorporaron estrategias de lectura pertinente para la comprensión lectora en sus tres niveles: literal, inferencial y crítico intertextual de textos expositivos, mediante actividades apoyadas con mapas conceptuales y organizadores de ideas, el uso de habilidades cognitivas como la observación, comparación, estructuración, ejemplificación, clasificación, interpretación y evaluación. El seguimiento a las secuencias se hizo mediante el diario de campo, instrumento donde se registró información sobre la práctica en el aula durante el desarrollo de las secuencias, convirtiéndose en una herramienta que ayudó a sintetizar las situaciones ocurridas, discriminar hechos relevantes y analizar los resultados.

\section{La comprensión lectora, el texto expositivo y las secuencias didácticas}

Existen diferentes teorías que determinan y describen las problemáticas de comprensión en el aula y en la sociedad colombiana, una de ellas es llamada déficit estratégico. Según Crespo (2004), este implica concebir y aplicar las estrategias metacognitivas necesarias para hacer de la lectura un proceso activo. Por ende, concebir también el texto expositivo como mecanismo de desarrollo que posibilita el perfeccionamiento cognoscitivo de los estudiantes a partir de la esquematización estructural de los contenidos, la interiorización de los conceptos y enunciados-argumentativos a partir de conclusiones individuales y agregados personales.

La comprensión lectora es una habilidad determinante que debe adquirir una persona desde que inicia su formación en la escuela. De las experiencias y estrategias con que un estudiante pueda contar para potenciarla dependerá su desempeño escolar, ya que le permitirá un empoderamiento eficaz del entorno social que lo rodea y las relaciones que establecerá con sus pares. Los desempeños mínimos e insatisfactorios en los resultados de las pruebas estandarizadas 
que se asocian a la falta de comprensión lectora en ambas instituciones, los escasos conocimientos previos, los factores afectivos, y motivacionales y los hábitos de estudio, generaron preguntas sobre las estrategias a utilizar para mejorar el desarrollo educativo de los estudiantes.

Se partió de las deficiencias en la comprensión lectora que presentaban los educandos para desentrañar el significado de los textos. En este sentido, era necesario, tal y como lo expresaba Álvarez (1996), considerar las características textuales, especialmente las marcas lingüísticas que constituyen esta clase de textos (micro y macroestructura), así como los otros tipos de texto: el narrativo, argumentativo, descriptivo y conversacional (p. 30).

El texto expositivo es una tipología textual que muestra información fática para intercambiarla de manera objetiva y evidenciar hechos reales, ideas propias o conceptos de contenidos, ya sean dirigidos a un grupo en específico o simplemente una temática idónea para desarrollar preparaciones puntuales. Poseen características y una estructura determinada que puede brindar estrategias para hacer posible el desarrollo de procesos cognoscitivos avanzados que ayudan a mejorar las habilidades de los estudiantes y por ende de las pruebas de estado.

Las prácticas en el aula posibilitan la presentación de la información de forma diferente, las cuales favorecen la ampliación del vocabulario, el conocimiento técnico y los referentes científicos. Su estricta organización busca un objetivo claro que funda la posibilidad de incrementar el conocimiento y la comprensión lectora por medio de una dirección enunciativa de información, que se determina en una sincronía de tiempo y espacio, y discrimina una introducción, un desarrollo y una conclusión que posibilita mentalmente la asimilación del mensaje y la interiorización con argumentos construidos desde la comprensión del individuo.

Para darle solidez conceptual a la propuesta, se revisaron experiencias como la de Luis Rico, De la Torre, Escolar y García (2016) en España, quienes realizaron un estudio acerca del proceso de la comprensión lectora expresiva a través de textos expositivos. Su objetivo fue mejorar la comprensión de textos continuos expositivos con el uso de las TIC. En México, Vega, Báñales, Reyna y Pérez (2014), analizaron la eficacia en el aprendizaje por medio del uso tres estrategias: 1) los patrones de organización; 2) los organizadores gráficos y 3) el resumen para mostrar la importancia de implementar el texto expositivo. En el ámbito nacional, el estudio de Murillo y Villa (2017) lo utilizaron como estrategia pedagógica desde el tópico del ciclo de vida de las plantas. Lo usaron para mejorar la comprensión de textos expositivos con estudiantes del grado tercero.

Según la investigadora Silva (2014), la comprensión lectora trasciende el conocimiento que la escuela imparte y va más allá de esta, pues da elementos 
para apropiarse del mundo de manera autónoma. De esta forma, en la investigación se abordó la comprensión lectora como esa habilidad necesaria para que el estudiante fuera más allá de la escuela y, por lo tanto, abarcara sus demás contextos para que pudiera enfrentarse ante diversas situaciones que se le presentaban y presentarán.

Una vez analizados los referentes teóricos, se llegó a la conclusión de que a través de las secuencias didácticas se intervendría en los grupos, ya que esta configuración didáctica permite identificar propósitos y condiciones tanto de inicio y desarrollo, como de cierre para evaluar y hacer seguimiento a los procesos y resultados involucrados.

Constituida por una serie de actividades desarrolladas de manera colectiva, la secuencia didáctica se puede realizar de forma individual o en pequeños grupos. Brindan la posibilidad de acceder a las diferentes nociones, habilidades y conocimientos, lo que permite que este tipo de configuración se convierta en una oportunidad de hacer partícipes al alumno y al docente en un proceso de enseñanza y aprendizaje. Esto es significativo en la medida en que se establecen conexiones entre los saberes y el contexto para dar solución a las actividades que están orientadas al alcance de los objetivos. Por lo tanto, la secuencia didáctica exige que en el aula el docente diseñe una serie de ejercicios que faciliten el trabajo, ya que el estudiante es parte activa, seleccionándolos y transformándolos en función de su uso en diferentes contextos (Dolz y Schneuwly, 2013).

Como cierre se utilizó el diario de campo como instrumento para centralizar la información del proceso; en esta bitácora de notas se puedo ampliar el panorama con dibujos, fotos y anexos que, catalogados de manera diáfana, hicieron que la información que se deseaba obtener en cada uno de los ejercicios planteados, permitiera profundizar sobre nuevos hechos en las situaciones que se atendieron, lo que dio secuencia al proceso de investigación e intervención, y disponer de datos para la posterior tarea evaluativa.

\section{Las secuencias didácticas diseñadas}

La población con la que contó la investigación estuvo conformada por veinte estudiantes pertenecientes a la Institución Educativa Compartir de San Antonio de Prado de la comuna ochenta y la Institución Educativa San Pablo de la comuna uno del grado quinto en edades entre los diez y doce años.

La Institución Educativa Compartir es de carácter urbano, mixta y con calendario A. Está ubicada en el corregimiento San Antonio de Prado, comuna ochenta, del municipio de Medellín. Los estratos socioeconómicos varían entre uno, dos y tres. Pasó de cobertura educativa contratada a institución de carácter oficial 
en el año 2017 bajo el modelo pedagógico desarrollista social. Alberga alrededor de mil estudiantes de educación formal en preescolar, básica primaria, básica secundaria, media académica y técnica en las jornadas: completa, mañana, tarde, nocturno y fines de semana. En la jornada de la mañana se encuentran los grados de sexto a once y uno de transición. En la tarde están los grados de primero a quinto y uno de transición. Cuenta con tres directivos y cuarenta y dos docentes. Se destaca en su misión al ofrecer educación inclusiva en todos los niveles mediante la apropiación de principios y valores, que permitan formar estudiantes competentes, críticos, reflexivos y con proyección comunitaria.

La Institución Educativa San Pablo está localizada en la comuna uno, zona nororiental del municipio de Medellín, donde el estrato socioeconómico oscila entre uno y dos. El contexto familiar es variable y la mayoría de las familias son de conformación monoparental o extensa. Se presenta un alto índice de desempleo, microtráfico, violencia intrafamiliar, segregación, machismo, entre otras características socioculturales. Esta institución está compuesta por la sede principal y dos secciones de preescolar, básica primaria, una de básica secundaria y una de media académica. En el barrio Moscú n. ${ }^{\circ} 2$ está la sección Marco Fidel Suárez, con cobertura aproximada de trescientos cincuenta alumnos, los cuales se distribuyen en cinco grupos de transición y cinco primeros. En la parte alta del barrio Villa de Guadalupe está la Escuela Medellín, cuya cobertura es de mil cien estudiantes con veintidós grupos de segundo a sexto grado; más dos grupos de aceleración y dos de brújula. La Institución Educativa San Pablo o antiguo Liceo San Pablo, está ubicada en el barrio del mismo nombre, con cobertura de mil cien estudiantes, con jornadas: mañana, tarde y nocturna. Cuenta con treinta y tres grupos de básica secundaria y media académica. Está compuesto por seis directivos, setenta y cinco docentes, tres profesionales de apoyo, dos para media técnica y dos docentes norteamericanos para la segunda lengua, más veinte personas de apoyo logístico y administrativo.

El modelo pedagógico adoptado por la institución es el social con enfoque cognitivo, el cual está enfocado en lograr el desarrollo del individuo con un proyecto de vida. Por lo tanto, dentro de la elaboración e implementación de las secuencias didácticas se integra el trabajo cooperativo como medio para organizar las actividades y fortalecer el trabajo conjunto en los estudiantes, el alcance de los objetivos y el aprendizaje, lo que se convierte en una experiencia social. Se asume entonces el trabajo cooperativo contemplado desde las competencias. Por ello, las secuencias didácticas no se enfocan únicamente a que los estudiantes aprendan determinados contenidos, sino que desarrollen competencias para desenvolverse en la vida, para lo que será necesario la apropiación de los contenidos en las diversas áreas (Tobón, Pimienta y García, 2010). 


\section{Secuencia didáctica uno}

Conociendo el texto expositivo diseñado para el área de lengua castellana, tuvo como propósito la comprensión y el reconocimiento del texto expositivo a nivel de función comunicativa, características y macroestructura por medio de un conjunto de actividades diseñadas para orientar el alcance del mismo. Iniciando con una actividad de motivación, los estudiantes fueron convocados por equipos a un juego de separación de párrafos, en el cual relacionaron subtítulos con los párrafos correctos. Luego del ejercicio, se realizaron preguntas de comprensión: ¿Cómo está organizado el texto? ¿Por qué son importantes los subtítulos? ¿La información presente en cada párrafo corresponde con los subtítulos? ¿Qué otros subtítulos usarían? ¿Qué otros textos han leído que estén organizados por párrafos? ¿Qué tipo de texto creen que es? Posteriormente se socializó para llegar a una confrontación grupal de opiniones y autoevaluación de sus actividades.

En la primera etapa se realizó una actividad con los párrafos y el paralelo con sus respectivos subtítulos. En la etapa dos se entregó a cada equipo un tipo de texto, con el cual debían sacar las palabras clave y analizar su estructura, además de explicar si era expositivo o narrativo y por qué. En la etapa tres se le concedió a cada equipo un tipo de texto narrativo o expositivo para identificar el tipo de texto; un representante de su equipo leía en voz alta mientras la maestra realizaba un paralelo con las características de ambos tipos de textos con los aportes de cada equipo.

\section{Secuencia didáctica dos}

Descubriendo textos expositivos se integraron las áreas de ciencias naturales, sociales, educación ambiental y lengua castellana. El objetivo de esta secuencia fue que los estudiantes reconocieran los diferentes tipos de textos expositivos (noticia, biografía y texto científico didáctico). Mediante esta secuencia se orientó el acercamiento no solo al reconocimiento de los textos mencionados anteriormente como clave en su inmersión al mundo social, sino que además, orientó procesos de comprensión lectora con cada uno de ellos de la siguiente manera:

En la primera etapa, como espacio introductorio se retroalimentaron los aspectos abordados en la secuencia anterior, cuya comprensión se observó en la gran mayoría de los estudiantes quienes también fueron medio para explicar y aclarar a quienes aún tenían dudas o vacíos conceptuales. En la segunda etapa se dio ambientación a la secuencia mediante la temática Piratas en búsqueda de los tesoros de los textos expositivos. Cada equipo cooperativo formó una tripulación y cada estudiante asumió un rol dentro de la misma, se presentó un mapa de los tesoros y se inició la búsqueda. Se realizaron ejercicios de comprensión lectora que involucraron los niveles y otros elementos conceptuales, 
por ejemplo, la estructura del texto mediante preguntas orientadoras. También se realizaron otras estrategias didácticas para su comprensión como los infogramas, considerados pistas para nombrar cada uno de los textos expositivos abordados. El primero de ellos fue la noticia sobre una enfermedad que afecta el sistema respiratorio. En la etapa tres se abordó el texto científico didáctico. Fue un ejercicio de lectura silenciosa, preguntas y luego ejercicio de lectura en voz alta. La etapa cuatro fue con la biografía, la etapa cinco con textos científicos y la etapa seis consistió en una prueba de comprensión lectora y reconocimiento de diferentes tipologías textuales.

\section{Secuencia didáctica tres}

Viajemos al mundo de un ecosistema diseñada para la interpretación de un texto expositivo. Tuvo como objetivo interpretar el texto a partir de los elementos de la superestructura: párrafo introductorio, párrafos de desarrollo, párrafo de finalización o conclusión y los marcadores descriptivos. También consistió en identificar las ideas principales en cada uno de los párrafos para la asignación de subtítulos, la intención comunicativa, el uso y significado de las imágenes presentes. Mediante el trabajo de un texto expositivo desde el área de ciencias naturales, se analizaron las diferentes estrategias que emplean los textos expositivos para su comprensión: definición, comparación, clasificación y estructuración.

\section{Secuencia didáctica cuatro}

La biografía de un gran líder mundial integró áreas como ciencias sociales, lengua castellana, ciencias naturales, educación ambiental y matemáticas. El objetivo principal fue la identificación de cómo se organizaban los textos expositivos con el fin de presentar información más clara y conocer la vida de diferentes líderes mundiales que lucharon por la paz e igualdad de derechos. Esta secuencia didáctica se llevó a cabo por medio de diferentes técnicas de organización de la información para la mejora de los procesos de comprensión.

Finalmente como fase cuatro de cierre y evaluación de las secuencias, se culminó el trabajo de intervención con una evaluación que contempló la comprensión lectora, la apropiación conceptual de los textos expositivos y la apreciación de los estudiantes entorno a las secuencias implementadas. De manera indiscriminada se entregaron cuatro pruebas de lectura con tipos de textos expositivos diferentes, uno por estudiante; cada uno de ellos contaba con un momento de lectura general, reconocimiento de las estrategias de comprensión al interior del texto, actividades de comprensión como subrayado de la idea principal, información nueva, desarrollo de organizadores gráficos, sub-titulación de párrafos, y preguntas de selección múltiple con única respuesta. 


\section{Conclusiones}

Esta investigación tuvo como objetivo general verificar la incidencia y el uso de los textos expositivos en el fortalecimiento de la compresión lectora con estudiantes de dos quintos de dos instituciones educativas públicas de la ciudad de Medellín. El problema fundamental de este estudio nació con dos situaciones que fueron visibilizadas en las prácticas de aula, y que se extendió al debate y cuestionamiento del quehacer docente.

Según los datos obtenidos en la caracterización del 100 \% de los estudiantes participantes, el $75 \%$ de ellos daban cuenta de un nivel de lectura literal, mientras que el $25 \%$ aún no se hacía evidente. El 47,5\% de los estudiantes se encontraba en un nivel de comprensión inferencial y el 52,5 \% no lo lograban. Solo un $27 \%$ demostraron un nivel de comprensión crítico intertextual, mientras que el $73 \%$ no demostraban dominio en este nivel.

La triangulación de los resultados de las técnicas utilizadas se realizó luego de explicar y hacer descripciones de los distintos ejercicios planteados para la recolección de datos, siendo necesario establecer algunas relaciones y comparaciones que se presentan aquí:

En los análisis de resultados de las técnicas de entrevista a docentes, estudiantes y la observación, se hallaron como punto en común la concepción sobre textos expositivos. Por un lado, algunos docentes presentaban dificultades sobre el concepto de texto expositivo, no sabían sus características. Además, estaban confundidos con su contenido e intención, pero los docentes que decían tener claridades, al momento de ser observados no dieron cuenta de la apropiación conceptual evidente en las prácticas de aula. Algunos maestros se acercaron al identificar los diferentes tipos de textos expositivos (históricos y periodísticos), sin que hubieran estrategias claras en su implementación didáctica.

La mayoría de los estudiantes, ejemplificó el texto expositivo con la noticia, como un medio para informarse de acontecimientos o hechos ocurridos, con lo que denotaron su visión limitada en cuanto al concepto, las características y la clasificación.

En cuanto a prácticas de aula con texto expositivo, se destacó la "motivación inicial" de la clase, los docentes realizaban una planificación que se hacía notoria en el desarrollo de la clase. Los estudiantes conocían el tema y los objetivos explicados al inicio de la sesión. Los docentes utilizaron como estrategia pedagógica el trabajo colaborativo y la asignación de roles; la indagación de saberes previos se llevó a cabo con un repaso de la clase anterior, recordando aspectos relevantes y contextualizados. 
En cuanto a la intención de los maestros en la utilización del texto expositivo, no se reconoció de manera clara, sino que se aproximaron a una intención en su uso, esto es, lo hicieron con el fin de informar para difundir un tema y un acercamiento a un concepto. En cuanto a la importancia del uso de texto expositivo en su área, la mayoría de ellos lo consideraron importante en todas las áreas del conocimiento, pero no expresaron claramente los beneficios al utilizarlo en todas las asignaturas, sencillamente fue usado como un tema o contenido propio del área de lengua castellana.

Algunos docentes tenían claras las prácticas para desarrollar las competencias en sus áreas (conocimientos previos, lecturas dirigida, proyección de videos, aclaración de conceptos, exposición de noticias, lluvia de ideas, diversas lecturas al texto), como estrategias para favorecer la comprensión de los diferentes textos, no solo expositivos. Asimismo, afirmaban que las lecturas de textos expositivos se debían implementar en todas las áreas, porque ayudaban a desarrollar competencias argumentativas e interpretativas y la crítica entre los estudiantes, además a transversalizar las áreas del conocimiento, y de ayudar a mejorar la comprensión lectora.

La investigación identificó que es necesario que los docentes se actualicen constantemente y acudan a los saberes previos, pues en ocasiones los estudiantes desconocían las funciones de los enunciados, lo que demostraba que los modelos tradicionales de enseñanza aportaban lo mínimo para que se identificaran los recursos textuales. Por lo tanto, es de vital importancia fundar componentes donde se consideren las cualidades de los contenidos y a partir de allí reforzar los saberes previos y posteriores. Los docentes mostraron que en momentos sus actividades son improvisadas con el texto expositivo, y el diseño de las actividades lo consideraban solo para reestructurar información objetiva o resumir una temática.

Al hacer referencia a las dificultades presentadas por los estudiantes al enfrentarse a un texto expositivo, los docentes mencionaron como una de las causas más importantes la falta de comprensión lectora, el vocabulario limitado que poseían y los niveles de análisis de los textos que se quedaban en lo literal.

Los estudiantes, en cuanto a las prácticas de aula, expresaron que se leían en clase noticias y se les daba mayor uso a textos narrativos. En cuanto a la lectura de texto expositivo, reconocieron su importancia pues era un recurso para informarse y encontrar descripciones de lo que deseaban aprender. Afirmaban que del texto informativo aprendían contenido verdadero, en este punto persistió la idea del texto expositivo informativo como único referente.

Los estudiantes, a su vez, para comprender los textos expositivos, con solo la lectura lograban entender. Las dificultades de lectura de estos textos las 
tuvieron con el vocabulario, por lo que expresaron la necesidad de una nueva explicación del docente para entenderlo. También afirmaron que el docente cuando explicaba nuevamente, cambiaba el método para mejor comprensión por medio de aclaraciones y formas de expresarlo.

En la triangulación de la teoría, la interpretación y los resultados, ofreció condiciones favorables para que los procesos de aprendizaje por medio del texto expositivo fueran de lo particular a lo general (títulos, encabezados y demás), con el fin de consolidar los procesos de concienciación de la información e identificación de los aspectos semánticos, dando cuenta de la información relevante que mejorara los procesos académicos y meta-cognitivos.

Con la orientación apropiada de los textos expositivos y la utilización de los ordenadores gráficos, los estudiantes avanzaron en los procesos de argumentación e identificación de la información relevante, manifestando desde su comprensión el contenido del texto; pero también les permitió contrastar la información, lo que ayudó a consolidar el nivel de lectura crítica.

Las inferencias que realizaron integraban conocimientos previos y argumentaciones que contribuyeron al mejoramiento de procesos de comprensión del mundo. El proceso alcanzó una mejora en los niveles de desempeño, en lo que respecta a la comprensión lectora. Además se identificó que es necesario innovar desde las planeaciones y estrategias de enseñanza las actividades con los textos expositivos, al igual que contextualizarlas de acuerdo a la edad, ritmos de aprendizaje y contexto social.

Con la utilización de mapas conceptuales y la identificación de información explícita, las habilidades de los estudiantes avanzaron en comparación, clasificación, deducción y jerarquización, fundamentales para las competencias en lenguaje y científicas.

La lectura literal mejoró y se alcanzó una transversalización de las diferentes áreas. Ello no desconoce que hay todavía dificultades en la identificación de tipologías textuales, pero alcanzar una relación textual ayudó a mejorar aprendizajes en las diversas áreas.

Las secuencias arrojaron resultados positivos, dado que el texto expositivo planteaba estrategias con sentido que ofrecían lógica a la información, visualizaron las características de los textos y discriminaron la información relevante logrando la comprensión necesaria. La jerarquización de las acciones y los textos expositivos se tornaron indispensables en el aula, y el diseño de secuencias didácticas fue un mecanismo para solución para las problemáticas de lectura.

En los diarios se comparó e identificó la necesidad de utilizar los textos expositivos y el aprendizaje cooperativo como manera de fortalecimiento en 
las estrategias de enseñanza, pues los procesos de aprendizaje mejoraron y la lectura cobró un sentido objetivo. La argumentación por medio del texto expositivo llevó a que los estudiantes se apropiaran de la información, la utilizaran y la analizaran relacionándola con la realidad que ofrece el contexto.

En el nivel inferencial los estudiantes presentaron ideas sobre el contenido del texto. El papel de la docente fue fundamental para plantear otras preguntas alternas a forma de contextualización, para que originaran otras respuestas acordes con lo que se preguntaba y para ello retomaron saberes previos para dar cuenta de los textos nuevos. En cuanto a la idea principal, hubo dificultad de reconocerla. Otros estudiantes asociaron la idea principal con una sola de las temáticas que desarrollaban los textos. No se identificaba la intención comunicativa del texto, lo que evidenció una lectura superficial y no dedujeron definiciones a partir de la ejemplificación de los textos.

En un ejercicio con el tema de las hormigas, todos hicieron una crítica correcta de las semejanzas presentes entre la vida en el hormiguero y las funciones de una familia. No se evidenciaron conocimientos de las hormigas por fuera del texto, confunden la función de las hormigas en su hormiguero y las relacionaron con sus imaginarios. No pudieron tomar una posición crítica sobre el texto, se les dificultó relacionar el texto leído con situaciones de contextos diferentes. Los estudiantes presentaron dificultades con el texto científico, pues es visto como un texto que informa y no como medio para tomar una postura crítica frente a este.

Al considerar lo expuesto, los procedimientos lingüísticos son necesarios para especificar objetivos de enfoque o tendencias para generar que las construcciones expositivas se coordinen, de tal manera que el estudiante pueda exponer al servicio del conocimiento, de los aprendizajes con claridad y de la información acorde a las realidades del ambiente, esto es, que respete la información ofrecida por los diferentes textos o aprendizajes. Pues el empleo de un léxico y una comprensión del mismo es fundamental para poder conquistar las ambigüedades de las interpretaciones y resignificar la utilización del texto expositivo como una posibilidad de transformar la educación memorística e ir a un valor temporal del argumento que no esté intervenido por la transmisión de mera información.

Se evidencia en el grado quinto deficiencias en la decodificación. Al mencionar algunas palabras (metamorfosis, larva, fértil, feromonas, estéril) incluso al marcar el acento prosódico u ortográfico, fue necesario que se repitiera la lectura de estas palabras y que el docente recordara su lectura correcta.

El texto no es visto como un mecanismo de crítica, sino como algo que proporciona información. En este sentido, es pertinente plantear a los estudiantes estrategias que les permitan visualizar características de lo que ya han leído y los aspectos a comparar, por ejemplo: hormiguero-familia, hormiguero-ecosistema. 
Una manera que puede conducir a que esto ocurra son los cuadros comparativos o esquemas jerárquicos, acciones que se deben pensar también desde la planificación inicial de una secuencia didáctica futura.

En las instituciones involucradas, se evidenció la escasez de prácticas reales con textos expositivos, pues la lectura y escritura se centraba en la utilización de textos narrativos que ofrecían información contada, en la que el sujeto debía esquematizar y discriminar lo relevante y así alcanzar, paso a paso, un nivel de lectura determinado. Se notó que las prácticas de lectura y escritura han sido desarrolladas de manera tradicional, donde se desconocen aspectos socioculturales o contextuales, sin tiempo y espacio que logre flexibilizar los procesos e incentivar los avances.

La escuela debe generar espacios alternos para las prácticas de lectura y escritura en horarios distintos a los del aula de clase. Como estos se constituyen en prácticas sociales e institucionales, importantes para consolidar los tejidos sociales, es importante hacer de estas prácticas socioculturales un ejercicio que redunde en la formación integral del estudiante.

Se deben reconocer los conocimientos y escenarios de los estudiantes sobre la escritura, para explicarlos y contextualizarlos examinando allí la importancia de la significación semántica de lo expuesto. Ya decía Emilia Ferreiro (1997) que desde el punto de vista constructivo, las escrituras infantiles evolucionan haciendo uso de los distintos medios culturales, las realidades educativas y del uso de diversas lenguas.

\section{Agradecimientos}

El presente artículo surge de la investigación realizada durante los años 2017 y 2018, titulada Prácticas de lectura con textos expositivos para mejorar la comprensión lectora en los estudiantes de grado $5^{\circ}$, de la Maestría en Educación de la Universidad de Medellín, en la línea de Didáctica de la Lectura y la Escritura de la facultad de Ciencias Sociales y Humanas. Beca otorgada por la Secretaría de Educación de Medellín.

\section{Referencias}

Álvarez, A. T. (1996). El texto expositivo-explicativo: su superestructura y características textuales. Revista Didáctica, (8), 29-44.

Crespo, F. A. (2004). El déficit estratégico y el fracaso escolar en los alumnos de la E.S.O. Conclusiones del I congreso anual sobre fracaso escolar 2004. Recuperado de http://www.fracasoescolar.com

Derechos Básicos de Aprendizaje DBA. (2015). Bogotá: Ministerio de Educación Nacional.

Dolz, J. y Schneuwly, B. (2013). Escribir es reescribir. Leer.es. Ministerio de Educación de España. Recuperado de https://leer.es/ 
Ferreiro, E. (1997). Alfabetización, teoría y práctica. México: Siglo XXI Editores.

Icfes. (2017). Pruebas saber. Recuperado de http://www2.icfesinteractivo.gov.co

Rico, L. De la Torre, T., Escolar, M. y García, J. (2016). Desarrollo de la competencia lectora expresiva mediante los textos expositivos. España: Universidad de Burgos. Recuperado de: http://www. redalyc.org/articulo.oa?id $=349851776044$

Ministerio de Educación Nacional. (2016). (G. 1. didácticas, Productor) de Red de maestros. Recuperado de http://aprende.colombiaaprende.edu.co/ckfinder/userfiles/files/LENGUAJE-GRADO-5.pdf

Murillo, C. y Villa, F. (2017). La Enseñanza para la comprensión como estrategia pedagógica para mejorar la comprensión de textos expositivos desde el tópico del ciclo de vida de las plantas (trabajo de grado). Universidad del Valle, Colombia.

Pérez, A. M. (1998). Evaluación de competencias en comprensión de textos ¿Qué evalúan las pruebas masivas en Colombia? Colombia: Universidad Pedagógica Nacional.

Silva, M. (2014). El estudio de la comprensión lectora en Latinoamérica: necesidad de un enfoque en la comprensión. Centro de Investigación Avanzada en Educación, Universidad de Chile.

Tobón, S., Pimienta, J., y García, J. (2010). Secuencias didácticas: aprendizaje y evaluación de competencias. México: Pearson.

Vega, N., Báñales, G., Reyna, A., y Pérez, E. (2014). Enseñanza de estrategias para la comprensión de textos expositivos con alumnos de sexto grado de primaria. Revista Mexicana de Investigación Educativa, (19), 47-68. Recuperado de: http://www.redalyc.org/articulo.oa?id=14032016003 